\title{
Active variable geometry suspension robust control for improved vehicle ride comfort and road holding
}

\author{
Cheng Cheng, Simos A. Evangelou, Carlos Arana and Daniele Dini
}

\begin{abstract}
This paper investigates the design of robust $\mathcal{H}_{\infty}$ control for road vehicle Series Active Variable Geometry Suspension (SAVGS). The objective is to improve ride comfort and road holding, while guaranteeing operation inside existing physical constraints. The study utilizes a nonlinear quarter car model that represents accurately the vertical dynamics and geometry of one quarter of a high performance car with a double wishbone suspension. The control objective is to reduce the body vertical acceleration, tire deflection and suspension travel under the impact of road perturbations. Therefore, the selection of the weighting functions for a linear $\mathcal{H}_{\infty}$ control, designed for the linearized quarter car, is based on these objectives. The proposed controller is then applied to the nonlinear quarter car model and investigated by nonlinear simulation for a range of road disturbance inputs. The results show that the designed controller when applied on the SAVGS is effective in improving the vehicle ride comfort and road holding.
\end{abstract}

\section{INTRODUCTION}

An important way to evaluate the performance of a suspension system of a road vehicle is to investigate its ability to offer good passenger ride comfort, vehicle road holding and to maintain overall vehicle stability. Over the past decades, research in suspensions has put much effort into reducing the conflict between achieving passenger comfort and road holding simultaneously [1]. Broadly speaking, depending on the nature of the suspension force element, three main types of suspension can be distinguished: passive, semi-active and active [2]. In relation to comfort and road holding, it is widely accepted that active suspension provides the best performance compared to conventional passive and semi-active suspensions. However, despite their advanced capabilities, active suspensions have not had a significant impact in the automotive market due to a number of disadvantages, related to power requirements, weight, size and complexity. The SAVGS used in the present study aims to provide performance that is better than passive and semi-active suspensions while avoiding the main disadvantages of active ones. The SAVGS has been proposed in [3] and its ability to control the vehicle low frequency dynamics, such as pitching and rolling motions under longitudinal and lateral acceleration, has been investigated in [4]-[6] with promising results. This paper focuses on the design of an SAVGS control system

C. Cheng (cheng.cheng12@imperial.ac.uk) and S. A. Evangelou (s. evangelou@imperial.ac.uk) are with Department of Electrical and Electronic Engineering, Imperial College London. C. Arana (carlos.aranaremirez10@imperial.ac.uk) is with the departments of Mechanical and Electrical and Electronic Engineering, Imperial College London. D. Dini (d.dini@imperial.ac.uk) is with the Department of Mechanical Engineering, Imperial College London. that tackles the higher frequency vehicle dynamics to provide improved ride comfort and road holding.

Various control strategies for active suspensions have been studied in the literature, such as $\mathcal{H}_{\infty}$ [7], Adaptive SlidingMode, Backstepping and Fuzzy Logic [8]-[11] control, typically by utilizing a model of one quarter of the car. In this paper, the aim is to design a linear $\mathcal{H}_{\infty}$ scheme to control a nonlinear quarter car model with SAVGS, representative of one corner of a high performance car with a double wishbone suspension arrangement. More precisely, the objective is to design control for the vertical acceleration, tire deflection and suspension travel using a linearized quarter car model, and then investigate the performance of the closed loop system by implementing the $\mathcal{H}_{\infty}$ controller to the nonlinear quarter car. Thus, a multi-objective $\mathcal{H}_{\infty}$ control strategy will result, which is able to provide an optimal road disturbance rejection to improve passenger ride comfort and road holding. At the same time, operating constraints of the suspension should be satisfied for a range of road disturbances.

This paper is organized as follows. Section II describes the model of the SAVGS and quarter car. Section III details the multi-objective $\mathcal{H}_{\infty}$ control synthesis. In Section IV simulation results of the passive and controlled nonlinear quarter car, for a range of road disturbances, are provided and compared. Conclusions are drawn in Section V.

\section{Modeling OF QUARTER CAR With SAVGS}

The SAVGS concept in single-link embodiment for a double-wishbone geometry, which is used in the present study, has been described in detail in [4]-[6]. A summary of its main characteristics is provided here. It extends a conventional passive double wishbone suspension arrangement by introducing a short rigid link between the upper end of the passive spring-damper unit and the chassis, as shown in Fig. 1. The joint of the single-link with the chassis is point $G$ and the joint of the single-link with the strut is point $F$. Any rotation of the single-link by the actuation torque $T_{S A V G S}$ modifies the spring-damper force and the suspension installation ratio. The single-link is generally rotated from the default equilibrium position (zero reference angle shown as the left hand side configuration in Fig. 1) to anything up to $180^{\circ}$. The actuator, which is fixed to the chassis, is a pair of servo motor and epicyclic gearbox that can be installed compactly in any space available adjacent to the single-link [5]. The SAVGS offers a number of advantages compared to other suspension types which include: (a) fail safe operation, (b) power regeneration capability, (c) no unsprung mass increase and small sprung mass increase, (d) use of 
existing well-developed electro-mechanical components, and (e) flexible packaging.
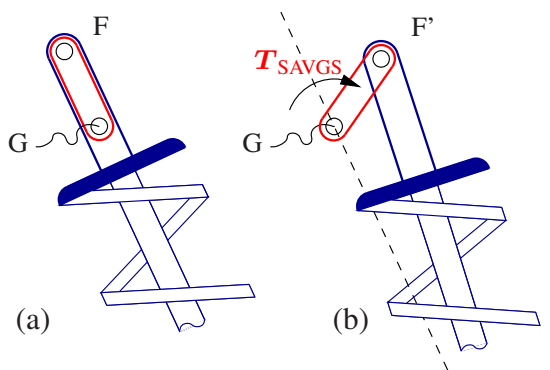

Fig. 1. Single-link variant of the SAVGS [5]. (a) Equilibrium position; (b) rotated (actuated) configuration.

The quarter car model used in this work extends the conventional quarter car to include a double wishbone arrangement as shown in Fig. 2. It involves a sprung mass

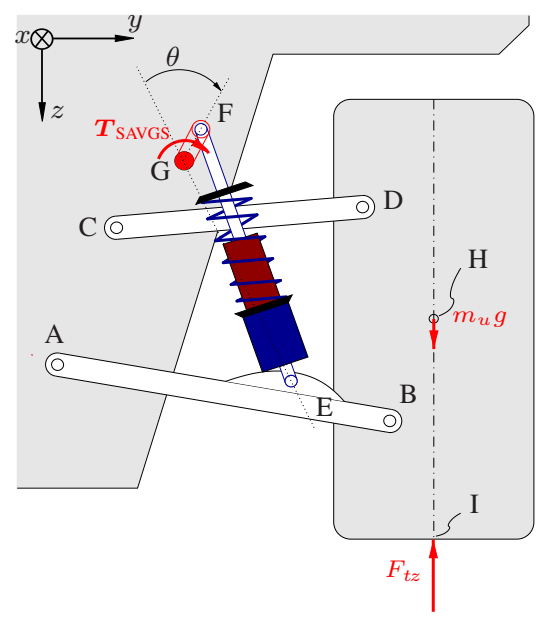

Fig. 2. Quarter-car arrangement. Points A, C and G are attached to the chassis; points $\mathrm{E}$ and $\mathrm{F}$ are fixed to the spring-damper unit; and points $\mathrm{B}$, $\mathrm{D}, \mathrm{H}$ and $\mathrm{I}$ are fixed to the wheel. The length of the spring-damper unit and its orientation can be continuously controlled through the rotation of the single-link with respect to its default equilibrium position [5].

(chassis) that is allowed to move vertically and an unsprung mass (wheel) that is connected to it via a massless double wishbone kinematic linkage. A road tire compression force $F_{t z}$ proportional to the tire deflection (the difference between the unsprung mass vertical displacement, $z_{t}$, and road input vertical displacement, $z_{r}$ ), acts on the unsprung mass to support the overall mass of the quarter car and to introduce the road forcing. A conventional suspension strut is combined with the new SAVGS components (single-link and actuator) and provide the force that suspends the sprung mass on the unsprung mass. Depending on the use of the quarter-car model the SAVGS part is added in one of two ways: (a) for linear analysis and control design, instead of using the torque $T_{S A V G S}$ as described above, the velocity of the single-link is used as an input and controlled directly, and no actuator dynamics are employed; this allows to reduce the complexity of the $\mathcal{H}_{\infty}$ control design prior to installing it on the full nonlinear model, and (b) for nonlinear simulation purposes the actuator dynamics are included with the plant and the actuator torque $T_{S A V G S}$ is generated by an inner control loop that aims to reduce the error between a singlelink angle input reference value and the actual single-link angle; the angle reference is calculated by integrating the output of the designed $\mathcal{H}_{\infty}$ controller.

The control schematic of the nonlinear simulation is shown in Fig. 3. There are two control loops involved, as introduced above. Firstly, $K_{s l p}$ is the position control scheme for the angle of the single-link. It makes use of saturation functions to ensure that operating and design constraints of the real system, such as the maximum single-link rotation angle and the maximum actuator torque, speed and power, are respected under any circumstances. This controller has been presented in [5] and the relevant schematic is shown here in Fig. 4. Secondly, $K_{\infty}$ is the $\mathcal{H}_{\infty}$ control scheme to be developed in this work which aims to reduce the body acceleration, tire deflection and suspension deflection (defined as the vertical component of the distance between the centers of mass of the sprung and unsprung masses) under external road disturbance forcing. The design procedure will be explained in detail in the next section. $\theta_{\text {ref }}^{*}$ is the reference rotational angle of the single-link, which is generated by the integration of the output from the $K_{\infty}$ block, and is subsequently tracked precisely by the inner position control loop. The saturation block in front of $\theta_{\text {ref }}^{*}$ is needed to ensure that the rotation of the single-link remains between chosen design boundaries $\left(0^{\circ}-180^{\circ}\right)$.

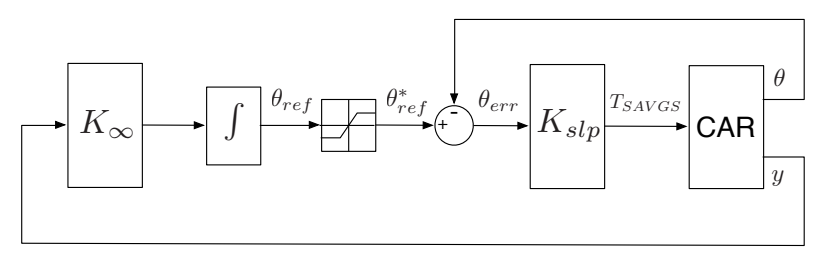

Fig. 3. Nonlinear control scheme for the quarter-car model.

The multibody modeling code AutoSim [12] is used to generate the overall quarter-car model. The AutoSim model can be used to a) generate $\mathrm{C}$ code containing the nonlinear equations of motion in order to run nonlinear standalone simulations or to produce a Simulink S-Function, or b) to generate symbolic state-space Matlab code that describes the small perturbation dynamics about a defined trim condition, for example at a non zero single-link angle equilibrium. The trim states can be found by nonlinear simulation and then used to evaluate numerically the state-space representation of the open-loop linearized equations of motion, for control design. The control design is performed in Matlab and tested by simulation in the Simulink environment.

\section{RoBUST CONTROL DESIGN}

The particular control purposes of the study presented here are to improve ride comfort and road holding. This is achieved by reducing the influence of road disturbances on body (sprung mass) acceleration and tire deflection respectively. The quarter-car model provides a suitable framework 


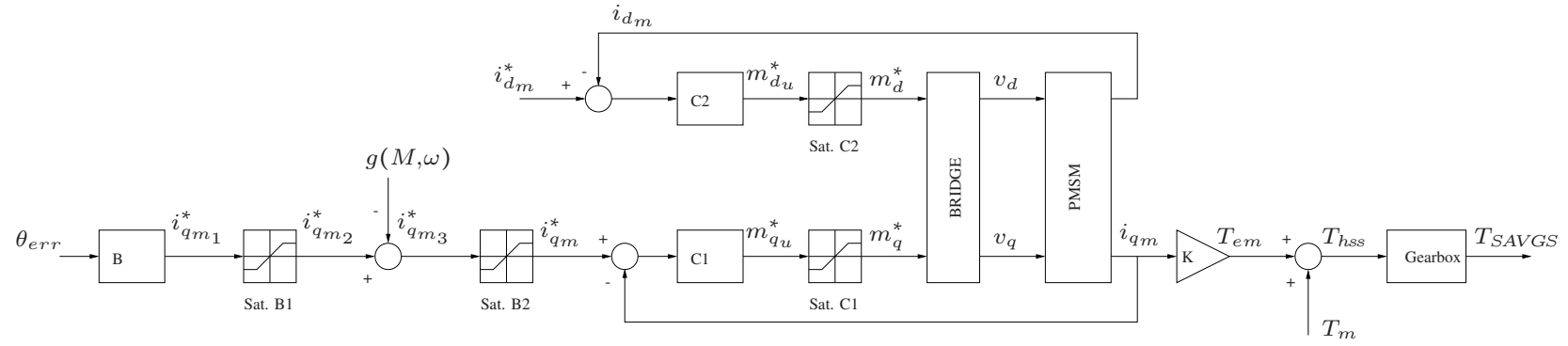

Fig. 4. Details of position control, $K_{s l p}$, of the single-link shown in Fig. 3. Starting from the position error signal, controllers B, C1 and C2 are combined with several dynamic saturation blocks and feedback loops in order to generate suitable voltage commands for the bridge converter that drives the PMSM actuator. The proposed scheme provides good position tracking performance and ensures that none of the physical or design limitations of the actuator are violated under any circumstances. Further details can be found in [5].

to study this behavior. The impact of road displacement input on suspension travel is also considered as an additional performance measure.

\section{A. $\mathcal{H}_{\infty}$ synthesis}

The $\mathcal{H}_{\infty}$ control problem is formulated according to the standard generalized regulator feedback structure [13] shown in Fig. 5. Although this figure shows a frequency-

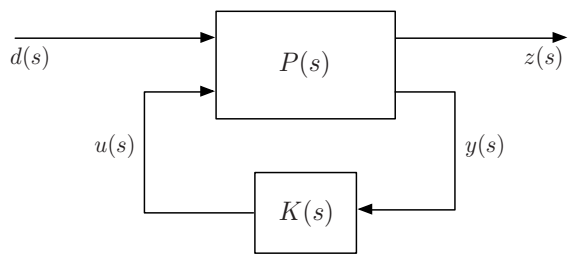

Fig. 5. $P(s)$ is the linearized quarter car model and $K(s)$ is the $\mathcal{H}_{\infty}$ controller. The signal $d(s)$ represents the vertical road displacement rate disturbance, $u(s)$ is the controlled single-link velocity input, $z(s)$ is the vector of three performance measure outputs (body acceleration, tire deflection, suspension deflection) and $y(s)$ is the suspension deflection rate used for feedback.

domain representation of the linearized system and feedback controller, it can also be adjusted for nonlinear time-domain studies, as shown in Fig. 3. In the following analysis, the Laplace variable $s$ is omitted from the various symbols and the use of the $\|\cdot\|_{\infty}$ is according to the standard definition [13]. With $P$ partitioned as

$$
P=\left[\begin{array}{ll}
P_{11} & P_{12} \\
P_{21} & P_{22}
\end{array}\right]
$$

the configuration of the generalized regulator is defined by

$$
\left[\begin{array}{l}
z \\
y
\end{array}\right]=\left[\begin{array}{ll}
P_{11} & P_{12} \\
P_{21} & P_{22}
\end{array}\right]\left[\begin{array}{l}
d \\
u
\end{array}\right]
$$

and

$$
u=K y
$$

which yields

$$
z=\left[P_{11}+P_{12} K\left[I-P_{22} K\right]^{-1} P_{21}\right] d .
$$

The aim is to design a controller $K$ that reduces the signal transmission path from road displacement rate forcing disturbances $d$ to performance outputs $z$ and also stabilizes the closed-loop system. Now consider the problem of finding $K$ which minimizes $\gamma$ such that

$$
\left\|P_{11}+P_{12} K\left[I-P_{22} K\right]^{-1} P_{21}\right\|_{\infty}<\gamma .
$$

By minimizing a suitably weighted version of (5) the control aim is achieved.

\section{B. Controller design}

For the purposes of control design the quarter-car equations of motion are linearized around a trim state of $90^{\circ}$ single-link angle to obtain $P$. The equilibrium state and equilibrium actuator torque are found by running a nonlinear simulation. At the trim condition selected the sensitivity of suspension force increment to single-link rotation is maximized and therefore the controllability of the system is maximized. It has also been shown previously [4]-[6] that at an offset angle of $90^{\circ}$ the SAVGS offers good control of the low frequency car dynamics. The state-space matrices of the linearized model $P$ are shown in the Appendix.

The design procedure involves the use of dynamic weighting functions to shape the plant transfer functions to meet the required design specifications. In the controller synthesis stage the plant transfer functions in Equation (5) are replaced with their frequency weighted versions. Figure 6 shows the control scheme structure and the weighting functions used. $W_{\text {perf } 1}$ and $W_{\text {perf } 2}$ are defined as low pass filters to penalize

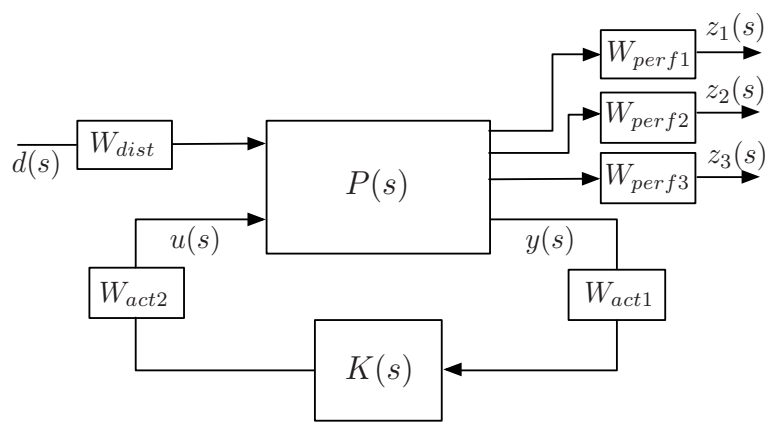

Fig. 6. $\mathcal{H}_{\infty}$ control scheme for disturbance rejection showing weighting functions, $W_{\text {act } 1}, W_{\text {act } 2}, W_{\text {dist }}, W_{\text {perf } 1}, W_{\text {perf } 2}$ and $W_{\text {perf } 3} \cdot z=$ $\left[\begin{array}{lll}z_{1} & z_{2} & z_{3}\end{array}\right]^{T}$ is the output vector with its elements corresponding to body vertical acceleration, tire deflection and suspension travel respectively. The other symbols have the same meaning as in Fig. 5. 
the appropriate low frequency ranges of body acceleration and tire deflection, corresponding to passenger comfort and road holding respectively. $W_{\text {perf } 3}$ is operating on the suspension travel to minimize it. A non-dynamic weight is found to be adequate, and since in the present design it is decided to give less significance to this objective, the weight is chosen to have a small constant value. $W_{a c t 1}$ and $W_{a c t 2}$ are used for 'loop shaping' and are finally absorbed into the controller such that the final controller is found by post-multiplying $W_{\text {act } 2} K W_{\text {act } 1}$. This means that the frequency properties of these two weights will be inherited by the controller. $W_{\text {act } 1}$ is chosen as a bandpass filter to concentrate the controller action in the frequency range of interest without expending much actuator power outside this range, while $W_{\text {act } 2}$ is given a constant value to keep the controller complexity low. A further design issue is the weight of the road disturbance input, $W_{d i s t}$, where it was found from trials that a nondynamic function is adequate. The final weighting functions are given as follows:

$$
\begin{aligned}
& W_{\text {perf } 1}=\frac{188.4(0.001 s+1)}{s+125.6}, W_{\text {perf } 2}=\frac{182.1(0.001 s+1)}{s+125.6}, \\
& W_{\text {act } 1}=\frac{3.5 \cdot 10^{6} s^{2}}{(s+5)(s+10)(s+100)(s+100)(s+100)}, \\
& W_{\text {act } 2}=0.01, \quad W_{\text {perf } 3}=0.01, \quad W_{\text {dist }}=1 .
\end{aligned}
$$

The optimization problem is defined in Matlab and the $\mathcal{H}_{\infty}$ problem is solved using the function hinfsyn. The controller that results has 16 states. Some iteration was involved in selecting the final weights by considering the performance of the linear closed-loop system and also by taking into account the resulting stability of the nonlinear closed loop system as found by simulation in the next section. The frequency response of the resulting controller is shown in Fig. 7. It can be seen in this figure that the controller has a 'bandpass' nature, as expected.

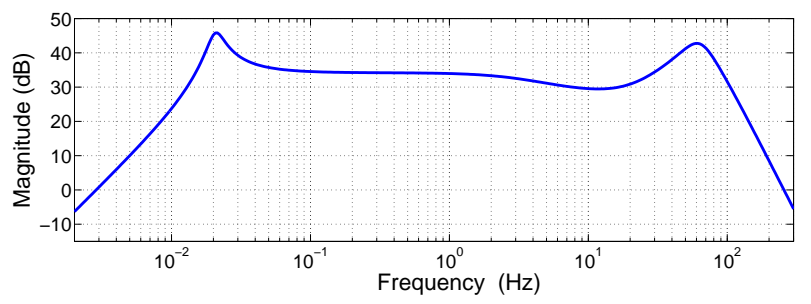

Fig. 7. Frequency response of the designed $\mathcal{H}_{\infty}$ controller.

\section{RESUlTS}

In this section the performance of the proposed $\mathcal{H}_{\infty}$ controller is tested by simulation, using the configuration shown in Fig. 3. The appropriate interaction of the $\mathcal{H}_{\infty}$ controller and the inner position control loop is critically important for the success of the overall scheme. This generally meant that the $\mathcal{H}_{\infty}$ controller required to be designed with its frequency response gain not as large as to saturate too heavily the response of the inner loop.
A controlled nonlinear quarter-car model associated with a high performance sports car is used in the simulation. The selected actuator comprises one Kollmorgen AKM33H, which is a Permanent Magnet Synchronous Motor (PMSM), and one Danaher UT075-40, which is an epicyclic gearbox. The key parameters for the car and SAVGS are shown in Tables I and II. The quarter car is run over a road surface with a) sinusoidal undulations $(0-30 \mathrm{~Hz})$, b) random road profile (ISO 8608:1995 [15]), and c) step disturbance, to cover a wide range of operating conditions. The offset angle of the single-link has been set at $90^{\circ}$ to correspond to the control design strategy in the previous section. For comparison purposes, results for the quarter-car equipped with purely passive suspension are also obtained. Since the control objective is to improve the ride comfort and road holding, the outputs selected and investigated in this section focus on the body vertical acceleration, tire deflection and suspension travel.

TABLE I

QUARTER-CAR VEHICLE PARAMETERS

\begin{tabular}{lcr}
\hline Parameter & Units & Value \\
\hline Total mass/Sprung mass & $\mathrm{kg}$ & $366 / 313$ \\
Spring stiffness & $\mathrm{N} / \mathrm{mm}$ & 158 \\
Tyre stiffness & $\mathrm{N} / \mathrm{mm}$ & 275 \\
Wheel radius & $\mathrm{mm}$ & 341 \\
\hline
\end{tabular}

TABLE II

SAVGS PARAMETERS

\begin{tabular}{lcr}
\hline Parameter & Units & Value \\
\hline Single-link length & $\mathrm{mm}$ & 11 \\
Offset angle & $\mathrm{deg}$ & 90 \\
Rotational limits & $\mathrm{rad}$ & {$[0, \pi]$} \\
Rated power (@160/320VDC) & $\mathrm{W}$ & $700 / 1310$ \\
Rated speed (@160/320VDC) & $\mathrm{rpm}$ & $2500 / 5500$ \\
DC bus voltage & $\mathrm{V}$ & 160 \\
Gear ratio & - & 40 \\
Total Mass & $\mathrm{kg}$ & $\sim 6$ \\
\hline
\end{tabular}

\section{A. Sinusoidal road forcing}

The nonlinear quarter-car, both in passive and active configuration, is forced by sinusoidal road inputs of amplitude $0.01 \mathrm{~m}$, and specified frequency in the range $0-30 \mathrm{~Hz}$, to examine and compare the frequency responses of the variables of interest. Each frequency response is found by taking the ratio of the output waveform rms value to the rms value of the input, at each frequency in the range of interest.

Frequency responses for the sprung-mass vertical acceleration, tire deflection and suspension travel, for the passive and SAVGS cases, are calculated and are shown in Fig. 8. It can be seen in all the three frequency responses associated with the passive system that the vertical dynamics of the vehicle exhibit the typical behavior for this vehicle category, in which the dominant effect is the existence of two resonant peaks at approximately $2 \mathrm{~Hz}$ and $10 \mathrm{~Hz}$, corresponding to sprung and unsprung mass resonances. The former peak falls 

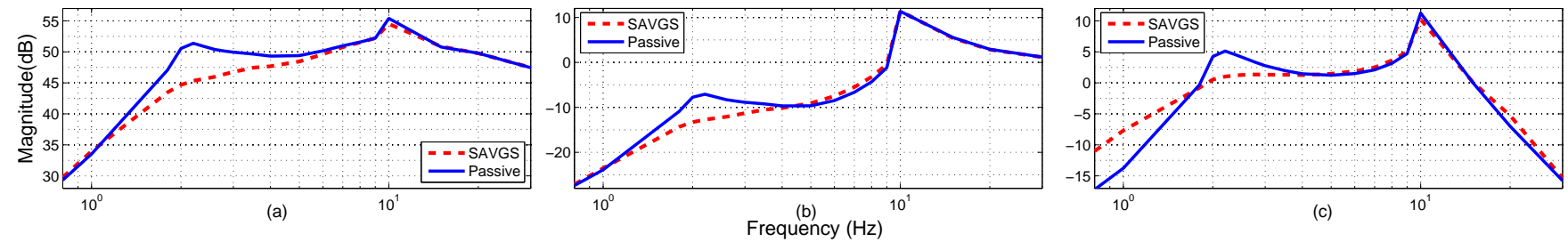

Fig. 8. Bode magnitude plots for the nonlinear quarter car model with sinusoidal road input of $0.01 \mathrm{~m}$ in amplitude and outputs of: a) body vertical acceleration with $0 \mathrm{~dB}$ corresponding to $\left.1\left(\mathrm{~m} / \mathrm{s}^{2}\right) / \mathrm{m}, \mathrm{b}\right)$ tire deflection with $0 \mathrm{~dB}$ corresponding to $1 \mathrm{~m} / \mathrm{m}$, and c) suspension travel with $0 \mathrm{~dB}$ corresponding to $1 \mathrm{~m} / \mathrm{m}$. The rms values of the inputs and outputs are used to calculate the gains.

in the range of frequencies where the human body is most sensitive to vertical acceleration (ISO 2361-1:1997 [14]). It is precisely in that frequency range that the $\mathcal{H}_{\infty}$ controller acts to reduce the vertical acceleration gain, as shown in Fig. 8 (a), to improve the ride comfort. The acceleration gain reduction achieved is up to approximately $6 \mathrm{~dB}$ at the resonant frequency near $2 \mathrm{~Hz}$. Fig. 8 (b) shows that road holding is also improved since the SAVGS achieves a reduction in the tire deflection gain at low frequencies $(1-4 \mathrm{~Hz})$ by up to $5.1 \mathrm{~dB}$. This allows the tire to run over the road with reduced variation in its normal load and therefore with improved grip. The frequency response presented in Fig. 8 (c) shows that the suspension travel is overall not adversely impacted while the controller is addressing the other two objectives. Its gain, as compared to the passive case, is even improved by up to $4 \mathrm{~dB}$ in the range of $1.7-4.4 \mathrm{~Hz}$, while at frequencies lower than $1.7 \mathrm{~Hz}$ its gain deteriorates, however the gains involved are much smaller than at higher frequencies.

Overall the sinusoidal road forcing results presented in this section demonstrate the positive performance of the SAVGS and $\mathcal{H}_{\infty}$ control scheme for the significant range of frequencies. Therefore the expectation is that the performance will be similarly improved in the case of more general road disturbances. This is investigated next.

\section{B. Random road forcing}

The generation of a random road profile to be studied here is based on the ISO 8608:1995 standard [15]. The road disturbance can be characterized in terms of its displacement power spectral density (PSD), in $\mathrm{m}^{2} /(\mathrm{rad} / \mathrm{m})$, which can be approximated to be [17]:

$$
\Phi(\omega)=\frac{\left(\sigma^{2} / \pi\right) \alpha V}{\omega^{2}+\alpha^{2} V^{2}},
$$

in which $\omega$ is the angular spatial frequency in $\mathrm{rad} / \mathrm{m}, \sigma^{2}$ is the road roughness variance in $\mathrm{m}^{2}, \mathrm{~V}$ is the forward velocity of the car in $\mathrm{m} / \mathrm{s}$, and $\alpha$ depends on the quality of the road and has units of $\mathrm{rad} \mathrm{s} / \mathrm{m}^{2}$. The road disturbance with the PSD in (6) can be produced by solving the differential equation

$$
\dot{z}_{r}(t)+\alpha V z_{r}(t)=w(t)
$$

in which $z_{r}$ is the road vertical displacement disturbance and $w$ is a white noise process with spectral density $2 \sigma^{2} \alpha V$. A typical highway road surface is generated by this expression in Simulink, with $V=30.6 \mathrm{~m} / \mathrm{s}, \alpha=0.127 \mathrm{rad} \mathrm{s} / \mathrm{m}^{2}$ and $\sigma=7.5 \times 10^{-3} \mathrm{~m}$. However, this road profile does not take into account the geometry of the tire. Hence, a rigid ring tire model with a fixed radius of $0.341 \mathrm{~m}$ is considered and a corresponding geometry filter is implemented to create a filtered version of the original road profile [16]. Fig. 9 depicts the final filtered random disturbance to be used as the road input.

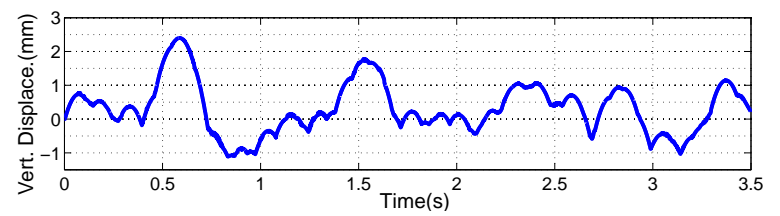

Fig. 9. Random road profile for $30.6 \mathrm{~m} / \mathrm{s}$ forward vehicle speed (the sign of vertical displacement is inverted for illustration purposes).

Nonlinear simulation results examining the performance of the quarter-car with SAVGS undergoing the random road disturbance are presented in Fig. 10. It can be seen in the
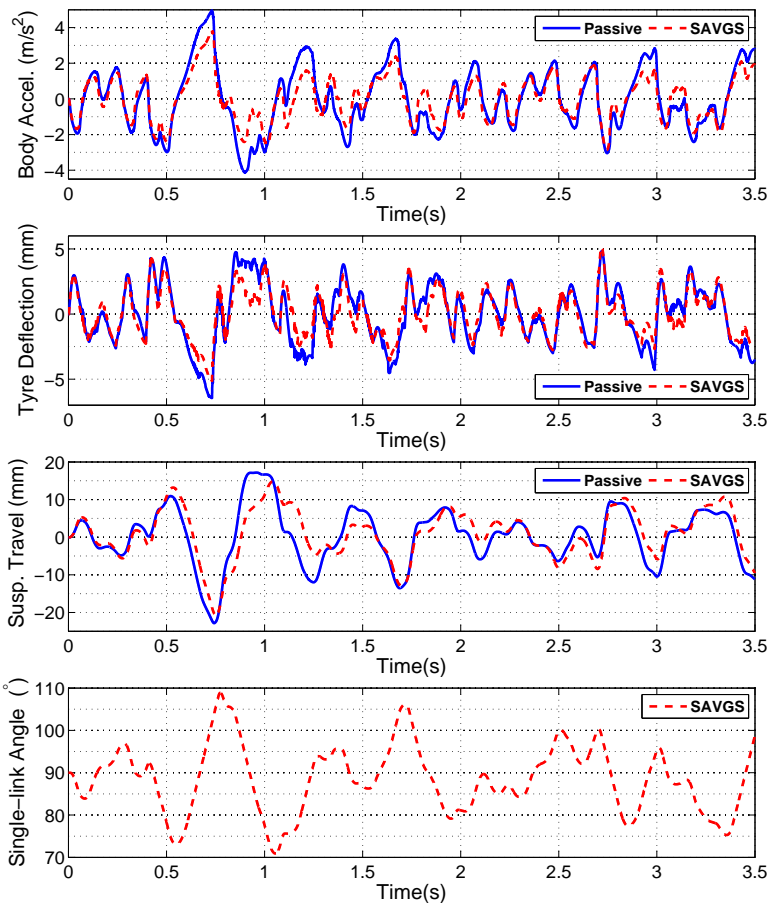

Fig. 10. Quarter-car time responses for the random road excitation in Fig. 9, for the passive suspension and SAVGS $\mathcal{H}_{\infty}$-controlled configurations.

first two plots that the body vertical acceleration and tire deflection have reduced peak values once the SAVGS with 
$\mathcal{H}_{\infty}$ control scheme is employed, as compared to the passive suspension case. This indicates the improved comfort and road holding properties of the active scheme. Also, the third plot in Fig. 10 illustrates that the maximum and minimum values of the suspension travel are not negatively affected by utilizing the active control scheme, while the final plot shows the corresponding usage of the single-link angle in the SAVGS. The time responses are now analyzed in more detail by making use of two standard fitness functions, the ride comfort and road holding indexes.

1) Ride comfort index (RCI): The ride comfort index is the rms bounce acceleration according to the ISO 26311:1997 standard [14], [18]:

$$
\mathrm{RCI}=\left[\frac{1}{T} \int_{0}^{T} a^{2}(t) \mathrm{d} t\right]^{\frac{1}{2}}
$$

in which $T$ is the vibration duration time and $a$ is the body vertical acceleration.

Table III presents results for the RCI and a further fitness function, the maximum vertical acceleration, for the responses in Fig. 10. The calculated RCI for the quartercar with SAVGS and $\mathcal{H}_{\infty}$ control is $27.7 \%$ less than for the passive system, and the maximum acceleration is similarly reduced by $22.4 \%$. These results imply the improvement in the ride comfort due to the implementation of SAVGS with the $\mathcal{H}_{\infty}$ control feedback loop.

TABLE III

RIDE COMFORT ANALYSIS (SI UNITS)

\begin{tabular}{lrrr}
\hline Fitness function & Passive & SAVGS & Improvement \\
\hline RCI & 1.77 & 1.28 & $27.7 \%$ \\
$\max (|a|)$ & 4.96 & 3.85 & $22.4 \%$ \\
\hline
\end{tabular}

2) Road holding index (RHI): The performance index for road holding is chosen to be the rms value of the tire deflection $z_{\text {def }}\left(=z_{t}-z_{r}\right)$ given by [20]:

$$
\mathrm{RHI}=\left[\frac{1}{T} \int_{0}^{T} z_{d e f}^{2}(t) \mathrm{d} t\right]^{\frac{1}{2}}
$$

Table IV presents results for the RHI and a further fitness function, the peak-to-peak tire deflection, for the tire deflection responses in Fig. 10. The RHI for the quarter-car with SAVGS and $\mathcal{H}_{\infty}$ control is reduced by $21.7 \%$ and the peakto-peak tire deflection is reduced by $8.8 \%$, as compared to the passive system. This demonstrates that the $\mathcal{H}_{\infty}$ controller can enhance the road holding ability of the car.

TABLE IV

ROAD HOLDING ANALYSIS (SI UNITS)

\begin{tabular}{lrrr}
\hline Fitness function & Passive & SAVGS & Improvement \\
\hline RHI & 0.0023 & 0.0018 & $21.7 \%$ \\
$\max \left(z_{\text {def }}\right)-\min \left(z_{\text {def }}\right)$ & 0.0113 & 0.0103 & $8.8 \%$ \\
\hline
\end{tabular}

\section{Step road forcing}

The final road perturbation used to evaluate the performance of the controller is a step change in the road height. In order to avoid numerical problems and to better represent the finite geometry of the tire, the step is smoothed and is given by the following expression in the time domain:

$$
f(t)= \begin{cases}\frac{h_{b}}{2}\left(1-\cos \left(\pi \frac{V}{w_{b}} t\right)\right) & 0 \leq t \leq \frac{w_{b}}{V} \\ h_{b} & t>\frac{w_{b}}{V}\end{cases}
$$

in which $h_{b}$ is the height of the bump and $w_{b}$ is is the longitudinal distance to rise from zero to the step height value. In the present case, moderate speed driving with $V=12 \mathrm{~m} / \mathrm{s}$ over a road step change with dimensions $h_{b}=0.05 \mathrm{~m}$ and $w_{b}=0.6 \mathrm{~m}$, is considered. Figure 11 presents the simulation results of the nonlinear passive and controlled systems. Compared with the passive system, the
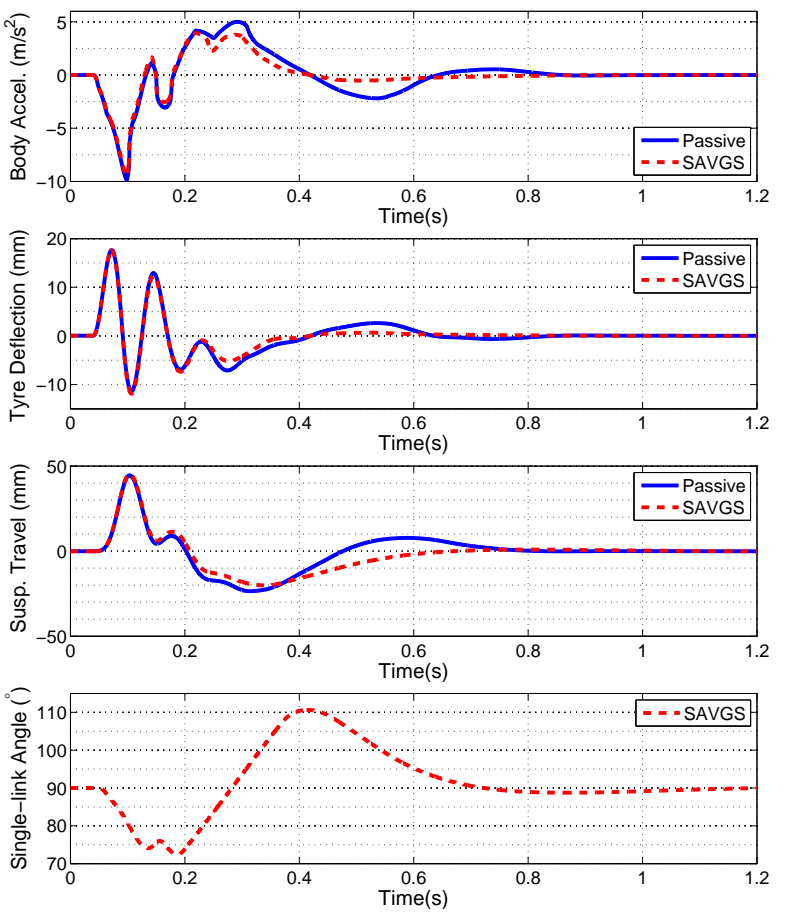

Fig. 11. Quarter-car time responses when running over a road bump of $0.05 \mathrm{~m}$ height at $12 \mathrm{~m} / \mathrm{s}$ forward vehicle speed, for the passive suspension and SAVGS $\mathcal{H}_{\infty}$-controlled configurations. The bump starts at $t=0.04 \mathrm{~s}$.

SAVGS $\mathcal{H}_{\infty}$-controlled system offers a good disturbance attenuation and quick response with short settling time, thus demonstrating once again its better comfort and road holding properties.

\section{Position controller performance}

The position controller for the single-link angle presented in Section II and Fig. 4 is designed to maintain operational limits to protect the PMSM and its servo-drive, to avoid damage of the gearbox, and to set the desired electric power consumption by the controller. In the present control scheme, the maximum output torque (on the low speed side of 
the gearbox) is conservatively limited to $97 \mathrm{Nm}$, which is the continuous limit for the selected actuator, and negative torques are not permitted in order to further reduce overall energy consumption. Thus it is possible to maintain the average power consumption below $35 \mathrm{~W}$ for the $2 \mathrm{~Hz}$ sinusoidal (a demanding sinusoidal case) and random road profile input cases. The single-link rotational speed is constrained within $\pm 13 \mathrm{rad} / \mathrm{s}$, and a soft constraint of $\pm 500 \mathrm{~W}$ is imposed on the electrical power flow from/to the PMSM.

Fig. 12 shows the evolution of output torque against single-link speed for the $2 \mathrm{~Hz}$ sinusoidal forcing, random excitation and step disturbance forcing road excitation events studied by simulation. It also shows the limit boundaries in actuator torque, speed and power. The figure clearly demonstrates that the operating points for all the simulations remain well within the design and operating constraints of the real system. Overall, the results show that the $\mathcal{H}_{\infty}$ controller scheme is able to improve the performance of the suspension while satisfying very stringent actuator performance limits. More aggressive control strategies for the same actuator will be explored in future investigations.

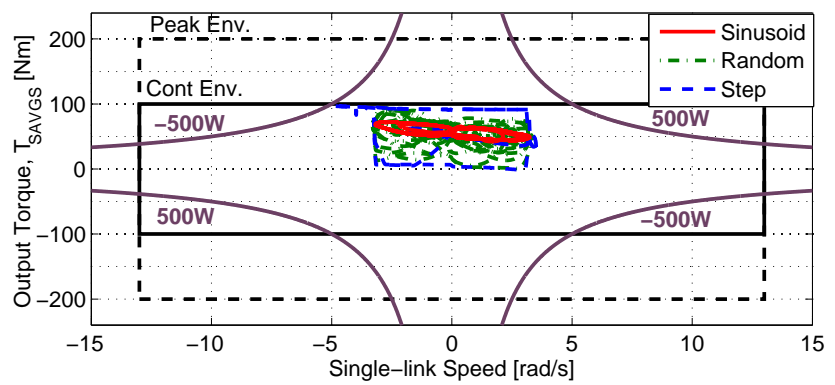

Fig. 12. Output torque vs. single-link speed characteristics for $2 \mathrm{~Hz}$ sinusoidal, random, and step disturbance forcing events of the nonlinear quarter-car with SAVGS, presented in Figs. 8, 10 and 11. The actuator limit boundaries are also shown.

\section{Conclusions}

Using a quarter-car model of a high performance car with double wishbone kinematic linkage and series active variable geometry suspension, a linear $\mathcal{H}_{\infty}$ control scheme is developed to reduce the impact of external road disturbances. Simulation results with the passive and nonlinear controlled system, for a range of road input disturbances, demonstrate that, as compared with a conventional passive suspension: a) the SAVGS with the proposed $\mathcal{H}_{\infty}$ controller offers a significant improvement in ride comfort and road holding of the vehicle, due to the reduction of body vertical acceleration and tire deflection, with limited impact on suspension travel, and b) the operation of the closed-loop system for realistic road conditions requires low power consumption and satisfies the torque and speed constraint characteristics of relatively small actuators, already available on the market.

\section{APPENDIX}

State-space matrices for the linearized quarter car model:

$$
A=\left[\begin{array}{cccc}
-2.186 & 11.81 & 0 & 0 \\
-11.81 & -2.186 & 0 & 0 \\
0 & 0 & -21.74 & 74.66 \\
0 & 0 & -74.66 & -21.74
\end{array}\right] \text {, }
$$

$$
\begin{gathered}
B=\left[\begin{array}{cc}
-11.1 & 0.201 \\
-36.35 & 0.676 \\
28.5 & 2.473 \\
230.6 & -0.07177
\end{array}\right], \\
C=\left[\begin{array}{cccc}
1.03 & -3.909 & 1.66 & -0.5674 \\
0.004206 & 0.02317 & 0.001072 & 0.003722 \\
-0.001184 & 0.004633 & -0.001256 & -0.003508 \\
-0.2827 & -0.0009847 & -0.3012 & -0.0008988
\end{array}\right], \\
D=\left[\begin{array}{cc}
0.01435 & -0.1152 \\
0 & 0 \\
0 & 0 \\
0 & 0
\end{array}\right] . \\
\text { REFERENCES }
\end{gathered}
$$

[1] D. Sammier, O. Sename, and L. Dugard, "Skyhook and h-infinity control of semi-active suspensions: Some practical aspects," Vehicle System Dynamics, vol. 39, no. 4, pp. 279-308, 2003.

[2] R. S. Sharp and D. A. Crolla, "Road vehicle suspension system design - a review," Vehicle System Dynamics, vol. 16, no. 3, pp. 167-192, 1987.

[3] S. A. Evangelou, D. Dini, O. D. Meerschman, A. Tocatlian, C.Kneip, and C. Palas, "Variable-geometry suspension apparatus and vehicle comprising such apparatus," Patent, 2012.

[4] C. Arana, S. A. Evangelou, and D. Dini, "Pitch angle reduction for cars under acceleration and braking by active variable geometry suspension," in Proc. 51st IEEE Conf. Decis. Contr., 2012, pp. 43904395.

[5] — "Series active variable geometry suspension for road vehicles." in IEEE/ASME Transactions on Mechatronics, vol. 20, no. 1, pp. 361$372,2015$.

[6] _ "Car attitude control by series mechatronic suspension," in 19th IFAC World congress on Automatic Control, 2014.

[7] H. Chen, P. Y. Sun, and K. H. Guo, "Constrained h-infinity control of active suspensions: an LMI approach," in International Conference on Control and Automation (ICCA), pp. 157-157, 2002.

[8] H. Li, J. Yu, C. Hilton, and H. Liu, "Adaptive sliding-mode control for nonlinear active suspension vehicle systems using fuzzy approach," IEEE Transactions on Industrial Electronics, vol. 60, no. 8, pp. 3328$3338,2013$.

[9] H. Du and N. Zhang, "Fuzzy control for nonlinear uncertain electrohydraulic active suspensions with input constraint," IEEE Transactions on Fuzzy Systems, vol. 17, no. 2, pp. 343-356, 2009.

[10] A. A. Basari, Y. M. Sam, and N. Hamzah, "Nonlinear active suspension system with backstepping control strategy," in IEEE Conference on Industrial Electronics and Applications, pp. 554-558, 2007.

[11] R. Rajamani and J. K. Hedrick, "Performance of active automotive suspensions with hydraulic actuators: theory and experiment," in American Control Conference (ACC), vol. 2, pp. 1214-1218, 1994.

[12] Anon. (1998) Autosim 2.5+ reference manual, mechanical simulation corporation, 709 west huron, ann arbor mi. [Online]. Available: http://www.carsim.com/

[13] M. Green and D. J. Limebeer, Linear robust control. Courier Dover Publications, 2012.

[14] "ISO 2631-1:1997, Evaluation of human exposure to whole-body vibration - Part 1: General requirements," 1997.

[15] "ISO 8608:1995, Mechanical Vibration-Road Surface Profiles- Reporting of Measured Data," 1995.

[16] P. W. A. Zegelaar, The dynamic response of tyres to brake torque variations and road unevennesses, Ph.D. Thesis, TU Delft, Netherlands, 1998.

[17] G. Corriga, S. Sanna, and G. Usai, "An optimal tandem activepassive suspension system for road vehicles with minimum power consumption," IEEE Transactions on Industrial Electronics, vol. 38, no. 3, pp. 210-216, 1991.

[18] M. Montazeri-Gh and M. Soleymani, "Genetic optimization of a fuzzy active suspension system based on human sensitivity to the transmitted vibrations," Proceedings of the Institution of Mechanical Engineers, Part D: Journal of Automobile Engineering, vol. 222, no. 10, pp. 1769-1780, 2008.

[19] "ISO 8855:2011, Road vehicles - Vehicle dynamics and road-holding ability," 2011.

[20] S. M. Savaresi, C. Poussot-Vassal, C. Spelta, O. Sename, and L. Dugard, Semi-Active Suspension Control Design for Vehicles. Elsevier Science, 2010. 